\title{
Prueba sensorial de grado de satisfacción para una ensalada y un dip tipo hummus elaborados a base de gandul (Cajanus cajan (L.) Mill sp.)
}

\author{
Carmen Andrés Jiménez ${ }^{1}$, Carolina Amerling Quesada ${ }^{2}$ \& Karol Saravia Zúñiga ${ }^{2}$ \\ 1. UNED.candres@uned.ac.cr \\ 2. UNED.camerling@uned.ac.cr \\ 3. UNED. ksaravia@uned.ac.cr
}

Recibido: 20 febrero 2013

Aceptado: 22 marzo 2013

\section{RESUMEN}

El gandul es una leguminosa de diferentes variedades que se está cultivando en la zona de San Carlos, Costa Rica, por parte de un grupo de productores con la asistencia de la UNED y el ICE. El desarrollo de productos nuevos a base de esta leguminosa se ha apoya por medio de la realización de pruebas sensoriales, entre las que se destaca la de grado de satisfacción de los panelistas, para la cual se utilizó una escala hedónica de 9 puntos y los comentarios que el panelista considerara conveniente sobre el producto. Se encontró que para ambos productos la aceptación fue de moderada ( $37.8 \%$ ensalada y $36.8 \%$ dip tipo hummus) a mucha (62.2\% ensalada y $63.2 \%$ dip tipo hummus). Además, se encontró que la apariencia de la ensalada y el sabor del dip tipo hummus fueron los atributos más aceptados por los panelistas, mientras que la cantidad de condimentos de la ensalada y la apariencia del dip tipo hummus fueron los más objetados. Se concluye que a un alto porcentaje de los panelistas ambos productos les gustó mucho y que hay características tales como el sabor y la apariencia, que se deben mejorar antes de ser lanzados al mercado. Se recomienda realizar pruebas a nivel piloto para mejorar la apariencia del dip tipo hummus y la cantidad de condimentos de la ensalada.

Palabras clave: gandul, prueba de grado de satisfacción, panel, panelistas.

\section{ABSTRACT}

Gandul is a legume of different varieties and it is being cultivated in San Carlos, Costa Rica by a group of farmers which is being supported by UNED and ICE. The development of new products based on this legume is being done by a kind of sensorial analysis called satisfaction level test, in which a 9 point hedonic scale was used along with the comments from panelists. For both products moderate (37.8\% salad y $36.8 \%$ hummus kind dip) to good acceptance was found (62.2\% salad y $63.2 \%$ hummus kind dip).
Furthermore, the appearance of the salad and the flavor of the hummus like dip were the most accepted attributes, while the amount of condiments of the salad and the appearance of the hummus kind dip were the ones panelists objected the most. It was concluded that a high percentage of the panelists liked both products very much (in the 9 point scale) and there are characteristics like flavor and appearance that need to be improved before launching the product to the market. It is recommended to do pilot plant tests to improve these attributes and correlate these results with different focus groups done for both products.

Key words: gandul, satisfaction level testing, panel, consumers.

\section{Introducción}

El gandul o comúnmente llamado frijol de palo, es una leguminosa originaria de la India, otros países de Asia y África, pero que se cultiva y comercializa en todo el mundo, en especial Estados Unidos, Puerto Rico, República Dominicana, Panamá y Perú (Ocampo, 2010, comunicación personal). En Costa Rica, aproximadamente hace cuatro años, se está cultivando en la zona de San Carlos por parte de la Asociación de productores de Gandul, raíces y tubérculos, ubicada en La Tigra del referido cantón.

El gandul cuenta con un alto valor nutricional, ya que contiene proteínas, minerales y vitaminas A y C (MAG, 1991), aunque también fibra y su sabor característico que goza de aceptación en otros países, ha sido aceptado por panelistas.

Debido a que el gandul es una vaina similar a los frijoles, en Costa Rica tiene el potencial de ser un cultivo alternativo, es decir, contribuye a 
la seguridad alimentaria del país. Por otro lado, su capacidad de fijar nitrógeno en el suelo y enriquecerlo con nutrientes, proteger las cuencas hidrográficas y ser abono para las plantas (Céspedes, 2012, comunicación personal); lo hace un cultivo muy beneficioso para el productor que lo adapte, ya que por sus características anatómicas de arbusto, se ha observado que es más resistente a las inclemencias del tiempo y por observación de los productores se ha comprobado que puede controlar ciertas plagas (Vega, 2012, comunicación personal).

Además de las características antes mencionadas, se ha comprobado a nivel de pruebas sensoriales por parte de las autoras, que es un producto muy versátil para la elaboración de diferentes productos como ensaladas, dip tipo hummus (Andrés, 2013) y guisos, entre otros.

Considerando lo antes expuesto, es que las autoras de este artículo desarrollaron dos productos elaborados a base de gandul, como lo son el dip tipo hummus y la ensalada.

Las ensaladas son platillos de consumo diario en los hogares costarricenses, mientras que el hummus es un producto alternativo para dip, wrap, sándwich y otros.

El hummus (palabra que en árabe significa "garbanzo"), es una ensalada o plato hecho a base de puré de garbanzos cocidos mezclados jugo de limón, tahina (pasta elaborada a base de semillas de sésamo tostadas) y aceite de oliva, que se procesa hasta obtener una pasta fina y que se le pueden adicionar otros ingredientes tales como paprika, ajo, chile picante, hojas de menta entre otros. Este plato se le considera de alto valor nutritivo por su contenido en hidratos de carbono, proteínas, vitaminas, minerales, fibra y grasas saludables. Cabe mencionar que gracias al aporte de vitamina $\mathrm{C}$ por parte de limón es que se absorbe mejor el hierro presente. Por lo general este producto se acompaña de pan árabe conocido como pan pita, aunque también se puede servir con vegetales cortados como zanahoria, pepino entre otros. Este platillo se considera muy popular en Oriente medio y en países como Israel, Líbano, Palestina, Turquía (VelSid, 2008).
La ensalada elaborada a base de gandul, es una ensalada con diferentes leguminosas, la cual es ideal para comercializarla en restaurantes y hoteles o para venderla empacada en supermercados en el área de productos listos para consumir.

La ensalada tiene ingredientes que ayudan a mantener su vida útil y le da un sabor, color, olor, textura y apariencia muy agradables al producto final.

Para los productos anteriormente mencionados, se decidió hacer un estudio de grado de satisfacción para obtener mayor información acerca del producto y generar datos menos subjetivos de los que se pueden obtener con una simple degustación. Esta prueba se debe realizar con panelistas no entrenados, ya que es necesario determinar el grado de satisfacción que muestran sobre el producto evaluado (AnzaldúaMorales, 1994).

Además, este estudio es un insumo para poder lanzar a mediano plazo este producto al mercado. Según Puldón, V; Suárez, E; Caraballo, R; Pachón, H \& Pompillo, C., 2011, al introducir un alimento al mercado o cambiar algún aspecto se requieren pruebas sensoriales al grupo al cual va dirigido el alimento (Puldón et al, 2011, p.3)

La evaluación sensorial que se realiza a los productos alimenticios como en este caso, se ocupa de la medición y cuantificación de las características de un producto, ingrediente o modelo, las cuales son percibidas por los sentidos humanos (Farfán, 2009, p.34).

Uno de los tipos de prueba sensorial utilizadas para tal efecto comprende los estudios de naturaleza hedónica, los cuales son esenciales para saber en qué medida un producto puede resultar agradable o desagradable al consumidor, además estas pueden ser aplicadas para conocer las primeras impresiones de un nuevo alimento o profundizar más y obtener información sobre su grado de aceptación. El mismo autor señala que los principales objetivos de un análisis sensorial basada en la escala hedónica son controlar la calidad de los productos ya existentes, identificar factores de especial importancia para el productor, conocer la aceptación de un nuevo producto 
en el mercado e intentar la mejora y optimización del mismo (Rabello y Lima, 2005).

Las escalas hedónicas de categorías consisten en una lista ordenada de posibles respuestas correspondientes a distintos grados de satisfacción, equilibradas alrededor de un punto neutro, donde el consumidor marca la respuesta que mejor refleja su opinión sobre el producto. Para la elaboración de la escala se considera la distribución subjetiva entre categorías, procurando ser estas equitativas. Estas pruebas están destinadas a valorar cuanto agrada o desagrada un producto y lo que se utiliza para tal efecto son escalas categorizadas que pueden tener diferentes niveles (Watts, 1992).

Los datos obtenidos de las pruebas sensoriales fueron evaluados o analizados para determinar si cumplían o no con una distribución normal. Para tal efecto, en paquetes estadísticos como SAS y SPSS se ejecuta el test de ShapiroWilk cuando el tamaño de la muestra es menor a 30 unidades y cuando es mayor, se aplica la prueba de Kolmogorov- Smirnov (Balluerka et. Al. 2002) y en ambos casos se llega a determinar un valor que al cotejarse contra una tabla me determina si los datos siguen o no la distribución normal y si esta es o no paramétrica (Delgado, 2004).

Posteriormente en un análisis estadístico se realizan los análisis de correlación que nos indica el grado de relación entre dos variables. En este sentido, el análisis de correlación de Spearman permite medir el grado hasta el cual los cambios en una variable están asociados con los cambios en la otra cuando se utilizan datos ordenados (Mc Daniel et al, 2005).

Por medio del análisis de cluster o conglomerados se puede medir si las variables están positivamente o negativamente correlacionadas o si son independientes. Este tipo de análisis comprende un grupo de técnicas multivariantes cuyo principal propósito es agrupar objetos basándose en las características que poseen y de esta manera clasificarlos de tal forma que cada objeto es muy parecido a los que hay en el conglomerado con respecto a algún criterio de selección determinado (Pedroza et al, 2006).
El objetivo de esta investigación fue realizar una medición de grado de satisfacción de una ensalada y un dip tipo hummus a base de gandul para obtener información objetiva del producto que permita mejorar la formulación de cada uno y determinar el nivel de aceptación que se podría dar en el mercado considerando que la materia prima principal es innovadora.

\section{Métodos}

Tomando como base determinado porcentaje de gandul mezclado con otras materias primas, se elaboraron una ensalada y un dip tipo hummus, los cuales fueron evaluados con una prueba de medición del grado de satisfacción por parte de 40 panelistas no entrenados, en la feria de proyectos de investigación realizada en la Escuela de Ciencias Exactas y Naturales el 14 de Noviembre, 2012.

Se utilizó una escala hedónica de 9 puntos (me gusta muchísimo-me disgusta muchísimo) y se realizó una validación estadística de los resultados obtenidos.

La metodología utilizada se detalla a continuación:

\section{Convocatoria a los participantes}

Durante la feria de proyectos de investigación de la Escuela de Ciencias Exactas y Naturales se contó con un stand de degustación en el que se motivó a las personas que se acercaban al lugar a degustar la ensalada y completar el formulario que contenía la escala hedónica de 9 puntos, además de escribir sus comentarios sobre el producto.

En total se contó con 40 participantes no entrenados, que se adapta a lo que AnzaldúaMorales, 1994, recomienda en relación con este tipo de pruebas.

\section{Definición de la formulación de producto}

\section{Ensalada}

Como materia prima principal se utilizó una mezcla de gandul de diferentes variedades (Catie, Criolla, Enana, Panameña y Sabalito) y 
los ingredientes secundarios utilizados fueron maíz dulce, garbanzos, guisantes, apio verde, cebolla morada, chile dulce, aceite de oliva, vinagre blanco, sal, paprika y pimienta negra.

La formulación del producto se diseñó de tal manera que el gandul fuera el ingrediente sobresaliente y el consumidor pudiera apreciar su sabor y colores para verlo como un producto nuevo (ver apéndice 2).

Los equipos industriales recomendados son: escaldador, balanza, cortadora, mezcladora, llenadora, empacadora, etiquetadora, entarimadora.

Se definió que el gandul es un producto que debe someterse a un proceso de remojo por al menos 12 horas, para posteriormente llevarlo a un proceso de cocción a un determinado grado de suavidad. El resto de las materias primas como maíz dulce, guisantes y garbanzos se utilizaron ya cocidos.

\section{Hummus}

Como materia prima principal se utilizó gandul de la variedad criolla y los ingredientes secundarios utilizados fueron jugo de limón, tahina, ajo, paprika, sal, hojas de menta y aceite de oliva.

Los equipos industriales recomendados son: desgranadora, escaldadora, molino de martillos, marmita, llenadora, selladora, etiquetadora, entarimadora.

Se definió que el gandul es un producto que debe someterse a un proceso de remojo por al menos 12 horas, para posteriormente llevarlo a un proceso de cocción a un determinado grado de suavidad. El resto de las materias primas se utilizaron ya procesadas.

\section{Preparación de muestras}

Ambos productos se elaboraron el día anterior a la feria, se mantuvo en refrigeración hasta la degustación y en el momento en el que se sometió a la prueba, se sirvieron, la ensalada en vasos plásticos con una cuchara plástica para que los panelistas pudieran realizar la prueba y el hummus en un vehículo como pan pita.

\section{Atributos a evaluar}

Para evaluar la ensalada se utilizaron los siguientes atributos, considerando lo que las autoras necesitaban saber sobre el producto: cantidad de sal, cantidad de condimentos, apariencia, acidez y sabor.

Para evaluar el hummus, se valoraron atributos tales como: textura, color, sabor, aroma y apariencia.

\section{Evaluación de atributos}

Para la evaluación de estos atributos, se utilizaron dos métodos: la escala hedónica (2.5.1) y los comentarios de los panelistas.

\section{Escala hedónica de 9 puntos}

En esta escala, el consumidor debe escoger entre 9 puntos que se muestran a continuación, según su criterio a la hora de degustar la muestra:

1 = Me gusta muchísimo

$2=$ Me gusta mucho

$3=$ Me gusta moderadamente

$4=$ Me gusta ligeramente

$5=$ No me gusta ni me disgusta

$6=$ Me gusta ligeramente

7 = Me gusta moderadamente

$8=$ Me gusta mucho

9 = Me gusta muchísimo

\section{Comentarios descriptivos al final de la prueba}

Para complementar la información obtenida en cada uno de los atributos evaluados, cada consumidor externaba un comentario general del producto si lo consideraba necesario y lo plasmaba en la hoja de respuestas (Apéndices 1 y 3).

\section{Análisis estadístico}

El proceso estadístico utilizado para analizar la información fue el siguiente:

\section{Prueba de normalidad}

Las pruebas de normalidad Shapiro-Wilk (n $<50$ ) y Kolmogorov-Smirnov se realizan para 
determinar si la información recopilada se considera estadísticamente normal o no, y así definir si las pruebas a utilizar son paramétricas o no.

\section{Correlación de Spearman}

La correlación de Spearman se aplica para determinar asociación entre los diferentes atributos evaluados (contenido de sal, apariencia, acidez, cantidad de condimentos y sabor).

\section{Análisis de cluster}

El análisis de cluster de dos fases se utiliza cuando una distribución de la información no es normal, y así determinar la cantidad de grupos en los cuales los panelistas se distribuyen y las características de cada uno de ellos.

\section{Resultados}

A continuación se muestran los resultados de la evaluación tanto del hummus como de la ensalada a base de gandul.

\section{Ensalada}

\section{Resultado general}

De manera general, el grado de satisfacción de la ensalada a base de gandul descrito por una escala hedónica de 9 puntos fue de 2.17 (me gusta mucho-me gusta moderadamente).

Además, para cada punto de la escala se obtuvo lo siguiente:

\section{CUADRO 1}

\section{Porcentaje de panelistas que escogieron cada uno de los puntos de la escala hedónica utilizada}

para realizar la prueba de grado de satisfacción de una ensalada a base de gandul

\begin{tabular}{lc}
\multicolumn{1}{c}{ Descriptor de la escala } & Porcentaje de panelistas que lo escogieron en promedio \\
Me gusta muchísimo & $40 \%$ \\
Me gusta mucho & $37.5 \%$ \\
Me gusta moderadamente & $7.5 \%$ \\
Me gusta poco & $15 \%$ \\
No me gusta ni me disgusta & 0 \\
Me disgusta poco & 0 \\
Me disgusta moderadamente & 0 \\
Me disgusta mucho & 0 \\
Me disgusta muchísimo & 0 \\
\hline
\end{tabular}

Fuente: Autoras, 2013 
En las siguientes figuras se muestran los resultados obtenidos para cada atributo evaluado de la ensalada de gandul:

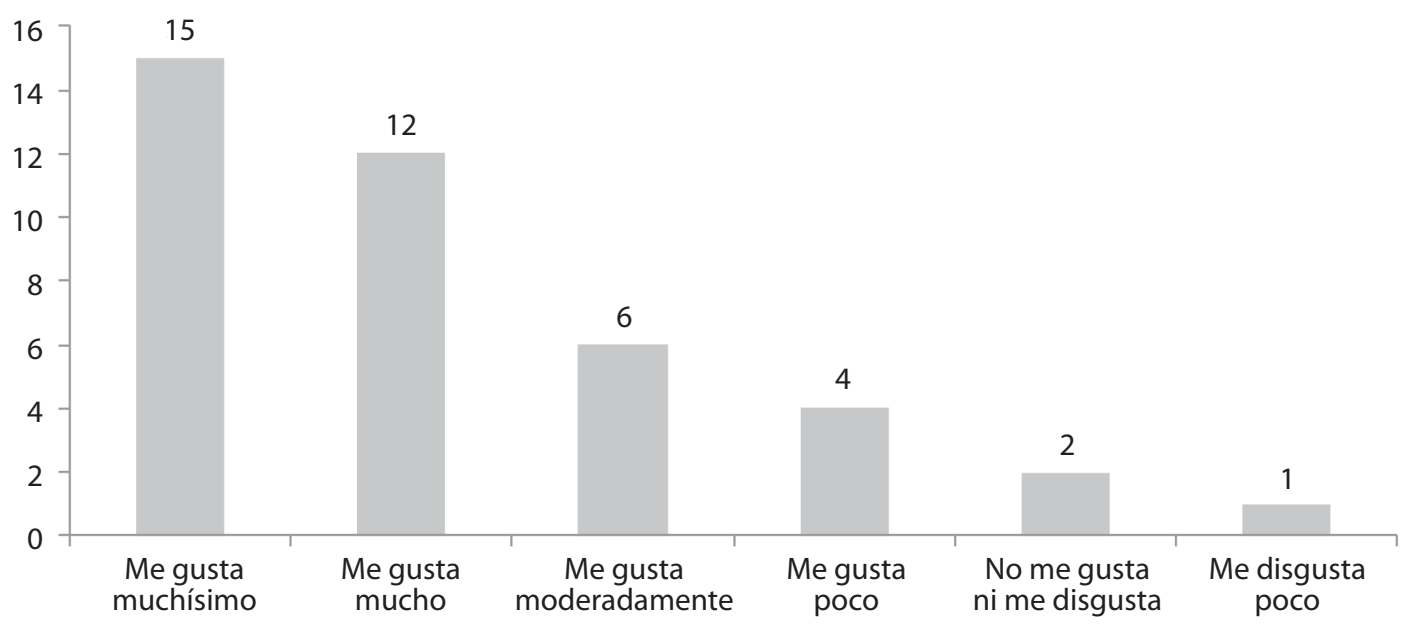

Figura 1. Grado de satisfacción del sabor de la ensalada de gandul evaluada por 40 panelistas. Mediana 2 (distribución no paramétrica). Fuente: Autoras, 2013.

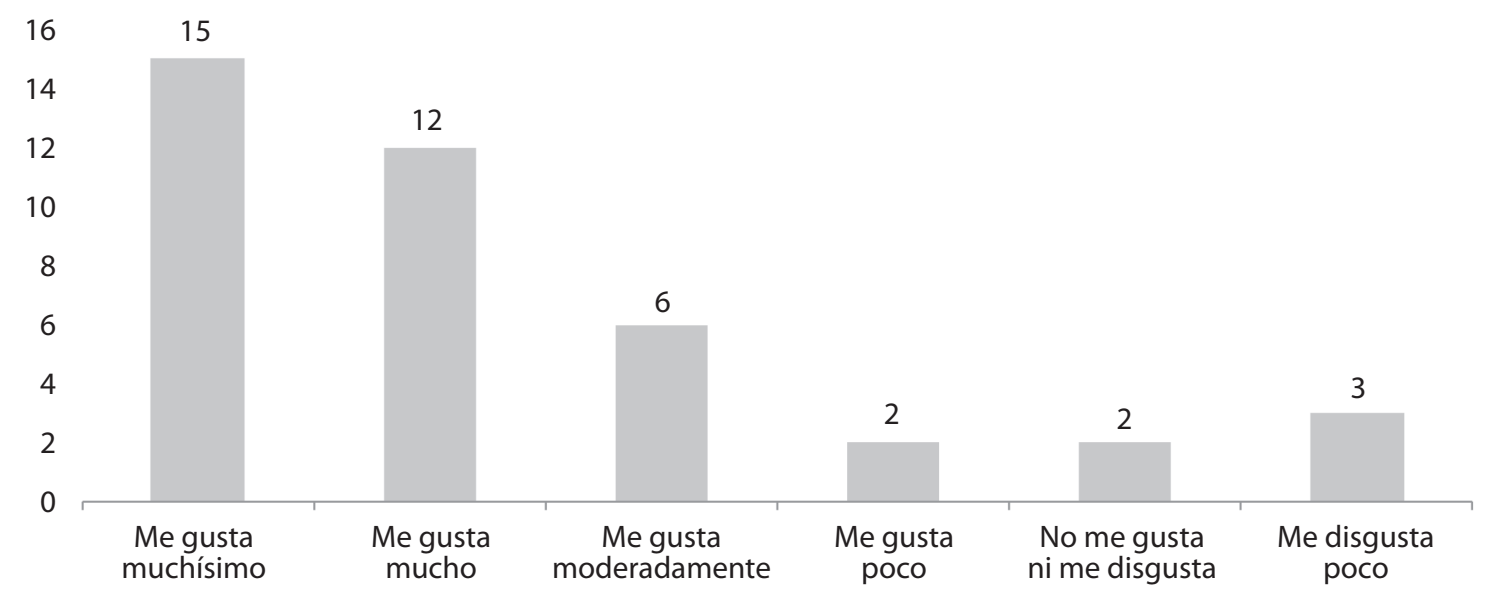

Figura 2. Grado de satisfacción de la acidez de la ensalada de gandul evaluada por 40 panelistas. Mediana 2 (distribución no paramétrica). Fuente: Autoras, 2013. 


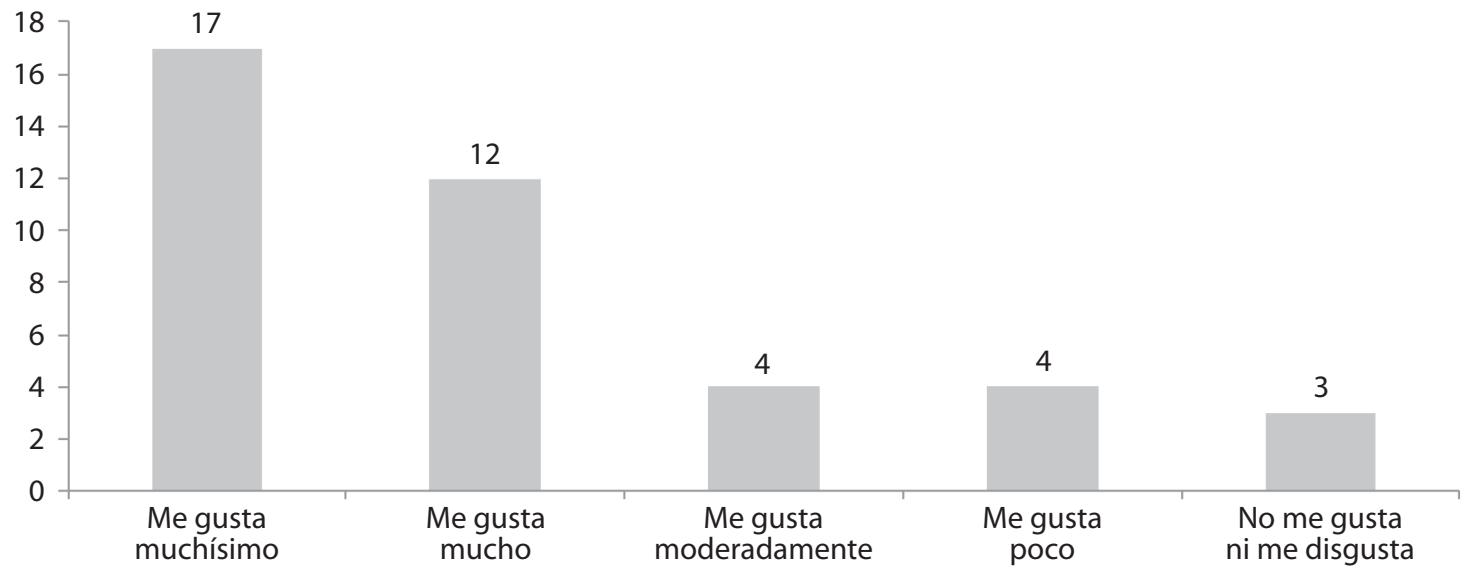

Figura 3. Grado de satisfacción de la cantidad de condimentos de la ensalada de gandul evaluada por 40 panelistas. Mediana 2 (distribución no paramétrica). Fuente: Autoras, 2013.

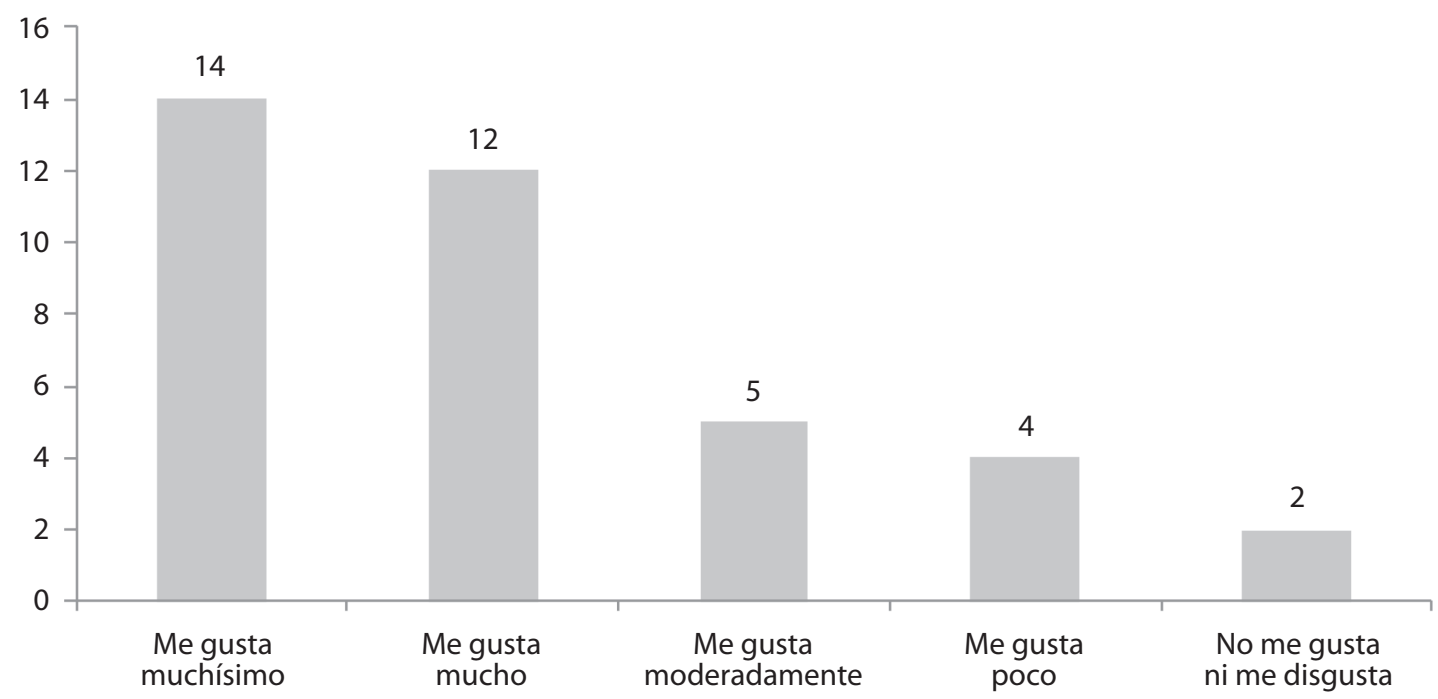

Figura 4. Grado de satisfacción de la cantidad de sal de la ensalada de gandul evaluada por 40 panelistas. Mediana 2 (distribución no paramétrica). Fuente: Autoras, 2013. 


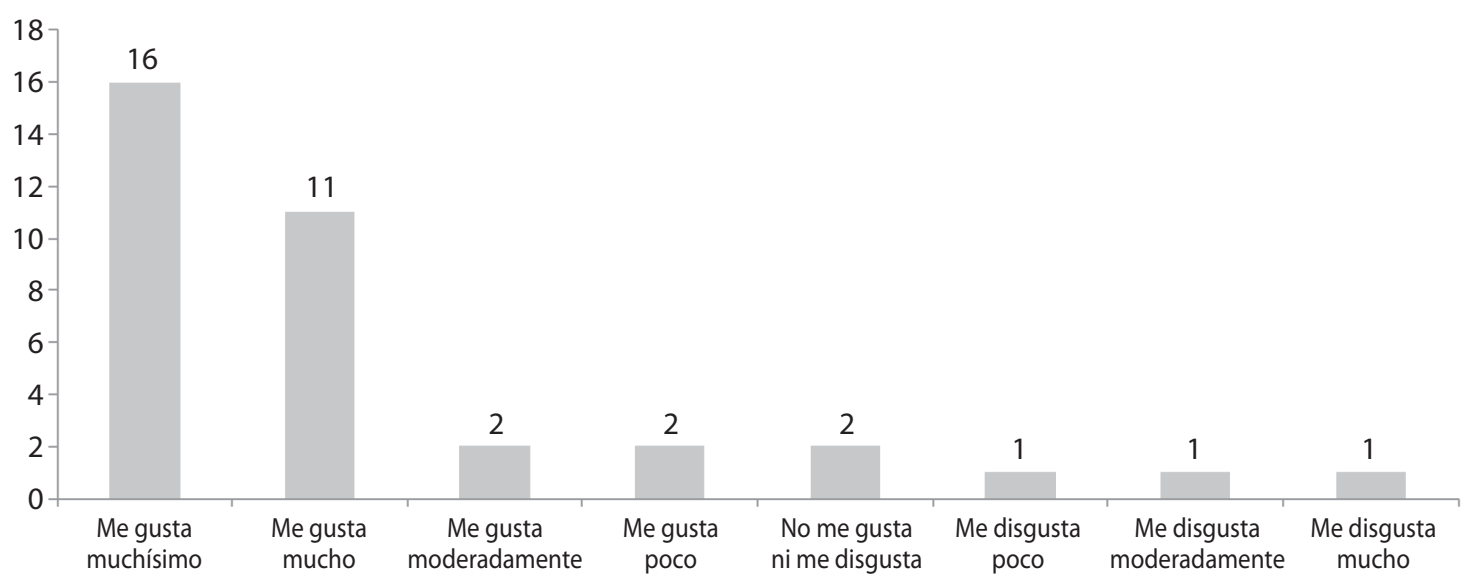

Figura 5. Grado de satisfacción de la cantidad de la apariencia de la ensalada de gandul evaluada por 40 panelistas. Mediana 1.5 (distribución no paramétrica). Fuente: Autoras, 2013.

\section{Análisis estadístico para la ensalada}

\section{a. Prueba de normalidad}

La prueba de normalidad de Kolmogorov-Smirnov es significativa, lo que indica que la distribución de las variables no es normal y la estadística no paramétrica es la que se debe utilizar.

\section{CUADRO 2}

\section{Prueba de normalidad para determinar la distribución de cada variable estudiada para en la prueba de grado de satisfacción de la ensalada a base de gandul}

\begin{tabular}{lccc} 
& \multicolumn{3}{c}{ Kolmogorov-Smirnov } \\
& Estadístico & gl & Sig \\
Sabor & .245 & 37 & .000 \\
Acidez & .252 & 37 & .000 \\
Apariencia & .310 & 37 & .000 \\
Condimentos & .252 & 37 & .000 \\
Sal & .247 & 37 & .000 \\
\hline
\end{tabular}

Fuente: Bermúdez, 2013

\section{b. Correlación de Spearman}

Dado que la distribución de los datos no es normal de los datos, se utilizó el coeficiente Rho de Spearman, el cual muestra que existe una correlación significativa entre los cinco factores analizados. La mayoría son altamente significativas ( $\mathrm{p}<0,01)$, y en dos casos significativas al $5 \%$. 


\section{CUADRO 3}

\section{Correlaciones de Spearman entre el contenido de sal, la acidez, el sabor, el contenido de condimentos y la apariencia de la ensalada a base de gandul evaluada con una prueba de grado de satisfacción}

\begin{tabular}{llrrrrr} 
& & Sabor & Acidez & Apariencia & Condimentos & Sal \\
Sabor & Coeficiente de correlación & 1.000 & $.515^{* *}$ & $.481^{* *}$ & $.700^{* *}$ & $.557^{* * *}$ \\
& Sig. (bilateral) & - & .001 & .002 & .000 & .000 \\
& $\mathrm{~N}$ & 40 & 40 & 40 & 40 & 37 \\
\multirow{5}{*}{ Acidez } & Coeficiente de correlación & $.515^{* *}$ & 1.000 & .351 & .666 & .503 \\
& Sig. (bilateral) & .001 & - & .026 & .000 & .001 \\
& $\mathrm{~N}$ & 40 & 40 & 40 & 40 & 37 \\
\multirow{5}{*}{ Apariencia } & Coeficiente de correlación & $.481^{* *}$ & $.351^{*}$ & 1.000 & .359 & .518 \\
& Sig. (bilateral) & .002 & .026 & - & .023 & .001 \\
& $\mathrm{~N}$ & 40 & 40 & 40 & 40 & 37 \\
\multirow{5}{*}{ Condimento } & Coeficiente de correlación & $.700^{* *}$ & $.666^{* *}$ & .359 & 1.000 & .589 \\
& Sig. (bilateral) & .000 & .000 & .023 & .000 \\
& $\mathrm{~N}$ & 40 & 40 & 40 & 40 & 37 \\
& Sal & $.557^{* *}$ & $.503^{* *}$ & .518 & .589 & 1.000 \\
& Coeficiente de correlación & .000 & .001 & .001 & .000 & - \\
& Sig. (bilateral) & 37 & 37 & 37 & 37 & 37 \\
\hline & $\mathrm{N}$ & & & & \\
\hline
\end{tabular}

* Correlación es significativa al nivel 0,01 (bilateral).

** Correlación es significativa al nivel 0,05 (bilateral).

Fuente: Bermúdez, 2013

\section{c. Análisis de cluster de dos fases}

- Calidad de los conglomerados

Resumen del modelo

\begin{tabular}{|l|c|}
\hline Algoritmo & Dos fases \\
\hline Características de entrada & 5 \\
\hline Agrupaciones & 2 \\
\hline
\end{tabular}

Calidad de conglomerados

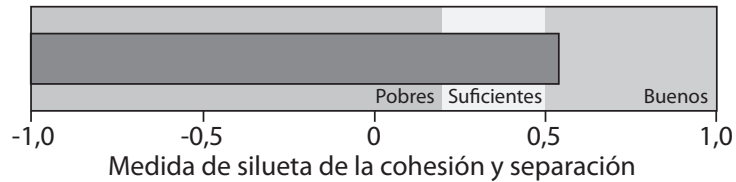

Figura 6. Calidad de los conglomerados generados para la evaluación del contenido de sal, la acidez, el sabor, el contenido de condimentos y la apariencia de la ensalada a base de gandul evaluada con una prueba de grado de satisfacción. Fuente: Bermúdez, 2013
- Tamaño de los conglomerados Tamaños de conglomerados

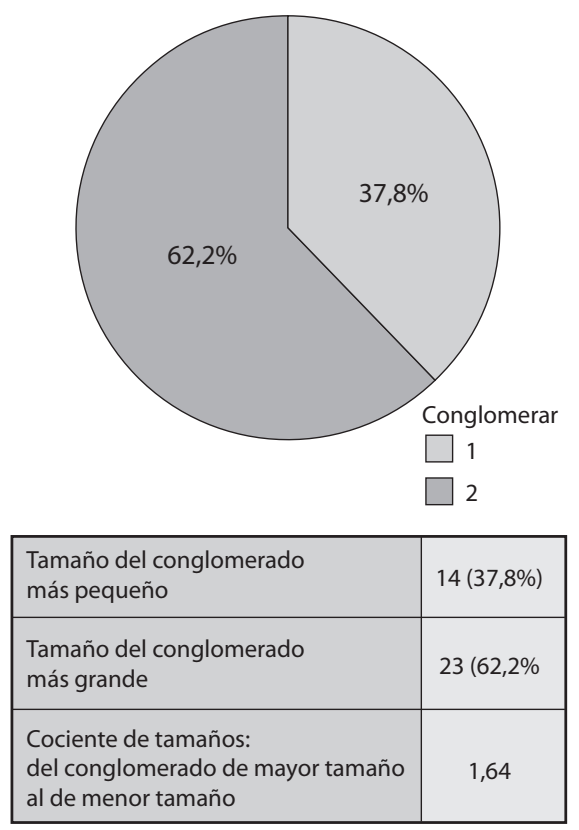

Figura 7. Porcentaje de panelistas que se encuentran en cada uno de los conglomerados formados estadísticamente para la prueba de aceptación realizada para una ensalada a base de gandul. Fuente: Bermúdez, 2013. 
Grupo 1: Les gusta moderadamente la ensalada $(38 \%)$

Es el grupo más pequeño, con un promedio global de 3,4 en la valoración de los cinco aspectos.

Grupo 2: Les gusta mucho la ensalada (62\%) En este grupo quedaron clasificados la mayoría de panelistas. El promedio general para las cinco variables es 1,5 .

- Variable de mayor precedencia

Con respecto a la variable de mayor precedencia para la clasificación es la valoración dada por los panelistas a los condimentos y la menor diferenciación corresponde a la apariencia.

\section{Agrupaciones}

Importancia de la característica

$\square 1,0 \square 0,9 \square 0,8 \square 0,7 \square 0,6 \square 0,5 \square 0,4$

\begin{tabular}{|c|c|c|}
\hline Conglomerar & 1 & 2 \\
\hline \multicolumn{3}{|l|}{ Etiqueta } \\
\hline \multicolumn{3}{|l|}{ Descripción } \\
\hline Tamaño & $\begin{array}{c}37,8 \% \\
(14)\end{array}$ & $\begin{array}{c}62,2 \% \\
(23)\end{array}$ \\
\hline \multirow[t]{5}{*}{ Funciones } & $\begin{array}{l}\text { Condimentos } \\
\qquad, 43\end{array}$ & $\begin{array}{c}\text { Condimentos } \\
1,39\end{array}$ \\
\hline & $\begin{array}{c}\text { Sabor } \\
3,50\end{array}$ & $\begin{array}{l}\text { Sabor } \\
1,61\end{array}$ \\
\hline & $\begin{array}{l}\text { Acidez } \\
3,71\end{array}$ & $\begin{array}{c}\text { Acidez } \\
1,61\end{array}$ \\
\hline & $\begin{array}{l}\text { Sal } \\
3,00\end{array}$ & $\begin{array}{c}\text { Sal } \\
1,61\end{array}$ \\
\hline & $\begin{array}{c}\text { Apariencia } \\
3,43\end{array}$ & $\begin{array}{c}\text { Apariencia } \\
1,52\end{array}$ \\
\hline
\end{tabular}

Figura 8. Variable de mayor precedencia para la clasificación de los panelistas que evaluaron la ensalada a base de gandul por medio de una prueba de grado de satisfacción. Fuente: Bermúdez, 2013.
Comentarios de los panelistas

CUADRO 4

Apreciaciones hechas por los panelistas sobre lo que les gustó y no les gustó de la ensalada a base de gandul de 5 variedades.

Lo que les gustó Lo que no les gustó

- El gandul por su sabor suavey . La cebolla (2 personas) textura (2 personas)

- Me gusta porque es diferente - La cantidad de grasa era mayor a lo que me gustaría (sabor residual a grasa) (3 personas)

- El utilizar productos nuevos - Acidez (2 personas)

- Combinación de ingredientes - No me gustó la textura del excelentes (2 personas) Gandul

- Sabor, aroma y apariencia - Hay muchos productos y el Gandul no se nota

- La apariencia es muy atractiva - Sabor muy fuerte a vinagre (2 personas)

- Sabor agradable y liviano - Incorporar otros vegetales

- Colores variados, mezcla (3 - Usar vinagre de uva personas)

- Lo ve como un sustituto de - Pasadito de aceite ensalada

- Producto saludable (2 personas) - Textura dura

- Apariencia agradable - Mucho aceite

- El sabor (7 personas) - Sabor un poco amargo

- Mezcla perfecta de sabores - El olor no me gustó

- La textura

Fuente: Autoras, 2013

Dip tipo hummus a base de gandul criollo

\section{Resultado general}

De manera general, el grado de satisfacción general del dip tipo humus a base de gandul descrito por una escala hedónica de 9 puntos fue de 2.47 (entre me gusta mucho y me gusta moderadamente).

Como se observa en el cuadro 5, para cada punto de la escala se obtuvieron los siguientes resultados: 
Porcentaje de panelistas que escogieron cada uno de los descriptores de la escala hedónica utilizada para realizar la prueba de grado de satisfacción del dip tipo hummus a base de gandul

Descriptor de la escala

Me gusta muchísimo

Me gusta mucho

Me gusta moderadamente

Me gusta poco

No me gusta ni me disgusta

Me disgusta poco

Me disgusta moderadamente

Me disgusta mucho

Me disgusta muchísimo
Porcentaje de panelistas que lo escogieron en promedio (\%)

30

36

17

12

2

2

0

0

0

Fuente: Autoras, 2013

Resultados de cada uno de los atributos del hummus evaluados

En las siguientes figuras se muestran los resultados obtenidos para cada atributo evaluado del dip tipo hummus a base de gandul:

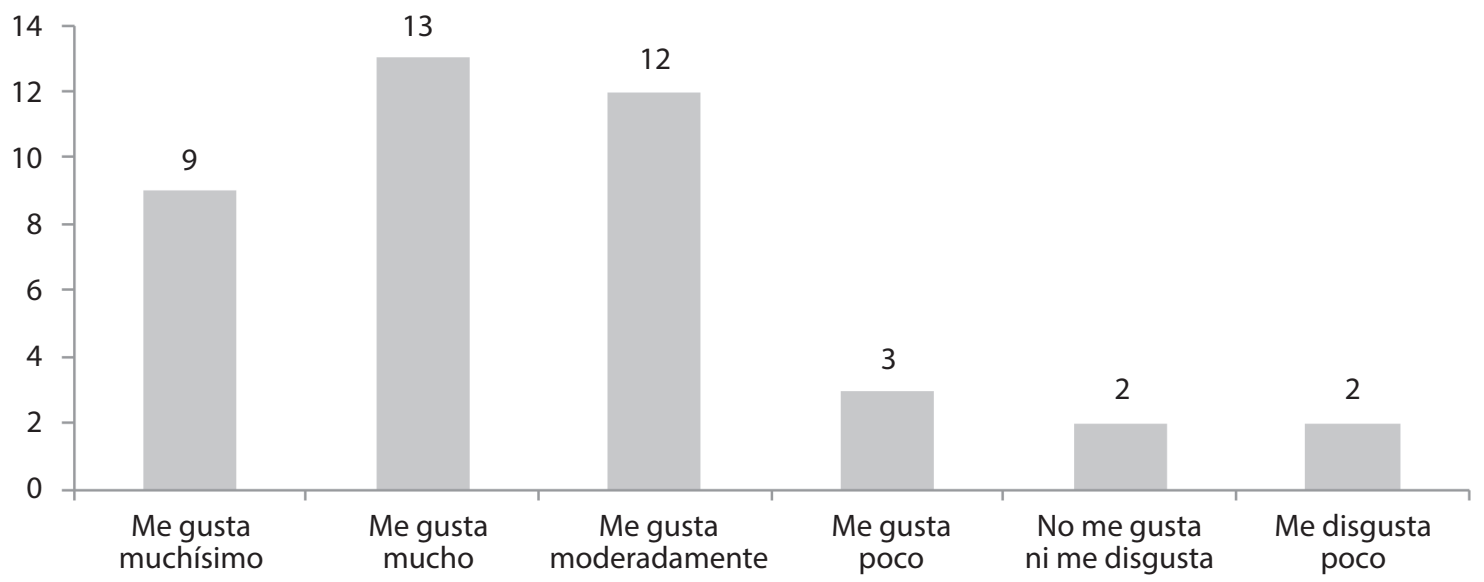

Figura 9. Grado de satisfacción del sabor del dip tipo hummus a base de gandul evaluada por 40 panelistas. Mediana 2 (estadística no paramétrica). Fuente: Autoras, 2013. 


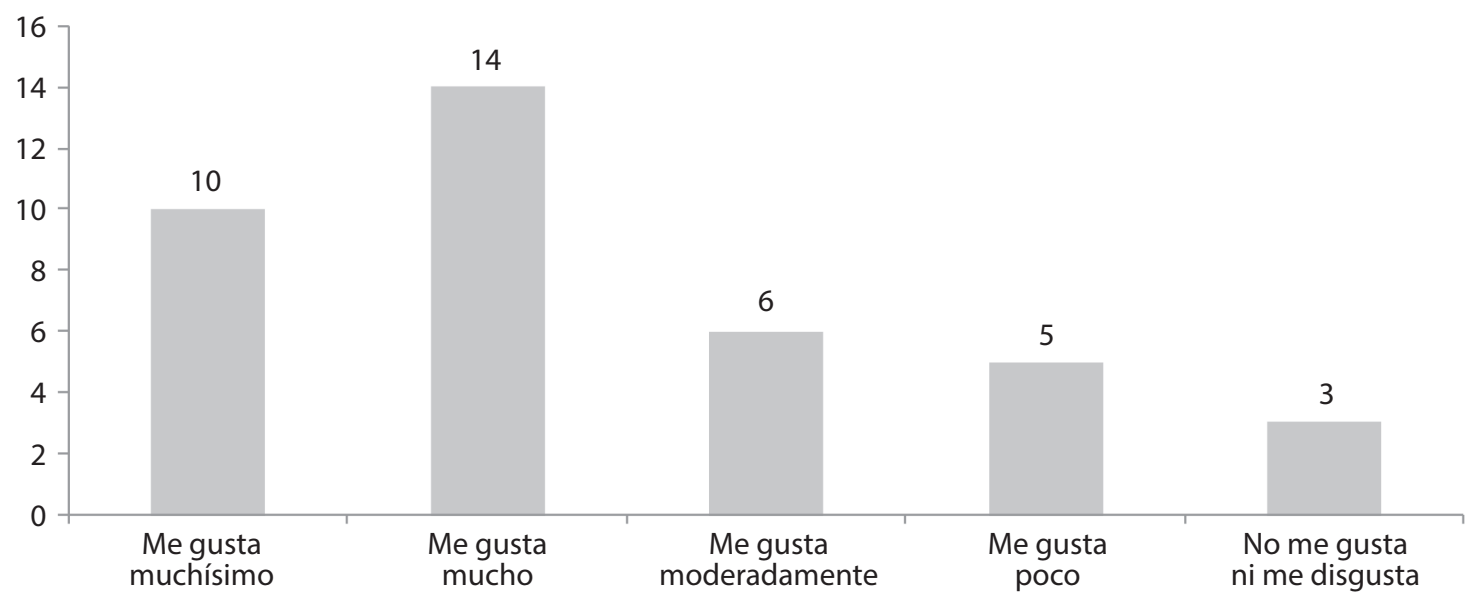

Figura 10. Grado de satisfacción del olor del dip tipo hummus a base de gandul evaluada por 40 panelistas. Mediana 2 (estadística no paramétrica). Fuente: Autoras, 2013.

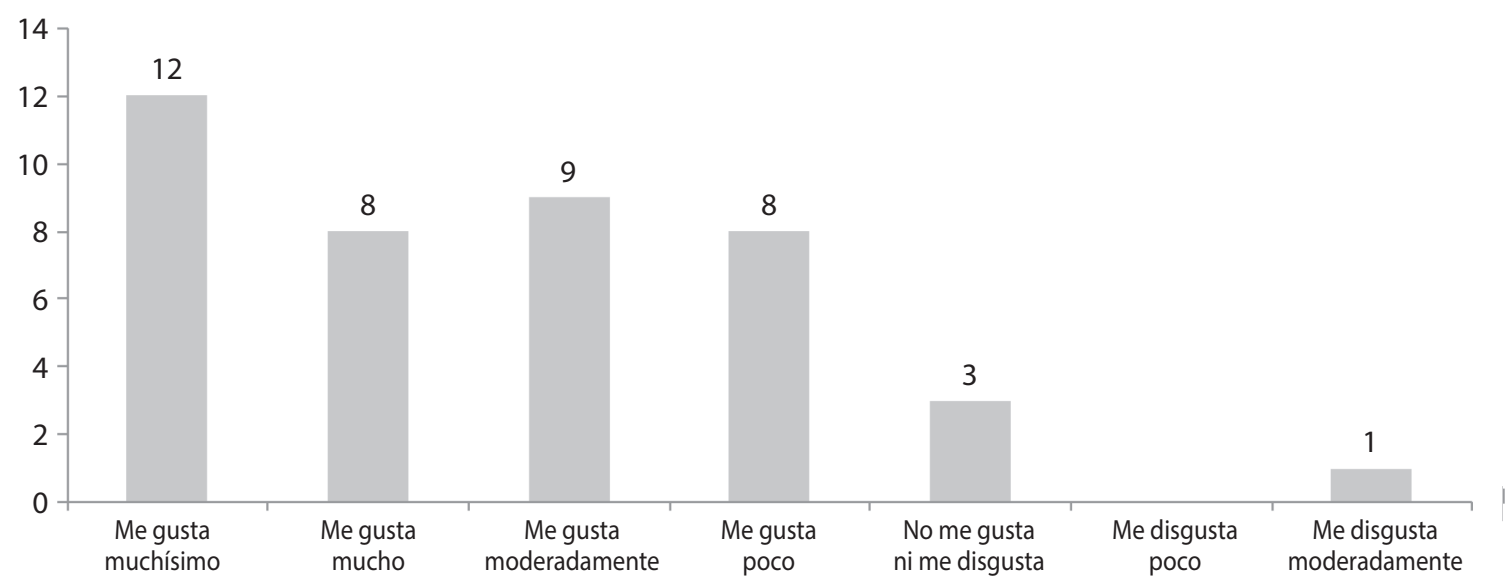

Figura 11. Grado de satisfacción de la apariencia del dip tipo hummus a base de gandul evaluada por 40 panelistas. Mediana 3 (estadística no paramétrica). Fuente: Autoras, 2013.

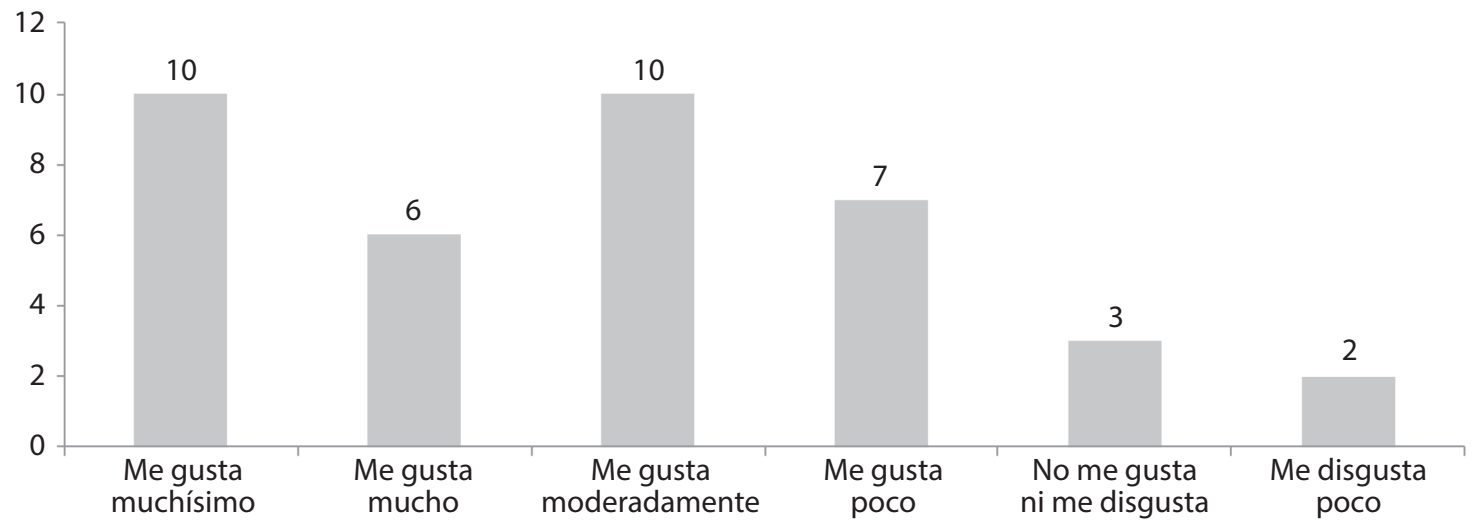

Figura 12. Grado de satisfacción de la cantidad del color del dip tipo hummus a base de gandul evaluada por 40 panelistas. Mediana 3 (estadística no paramétrica). Fuente: Autoras, 2013. 


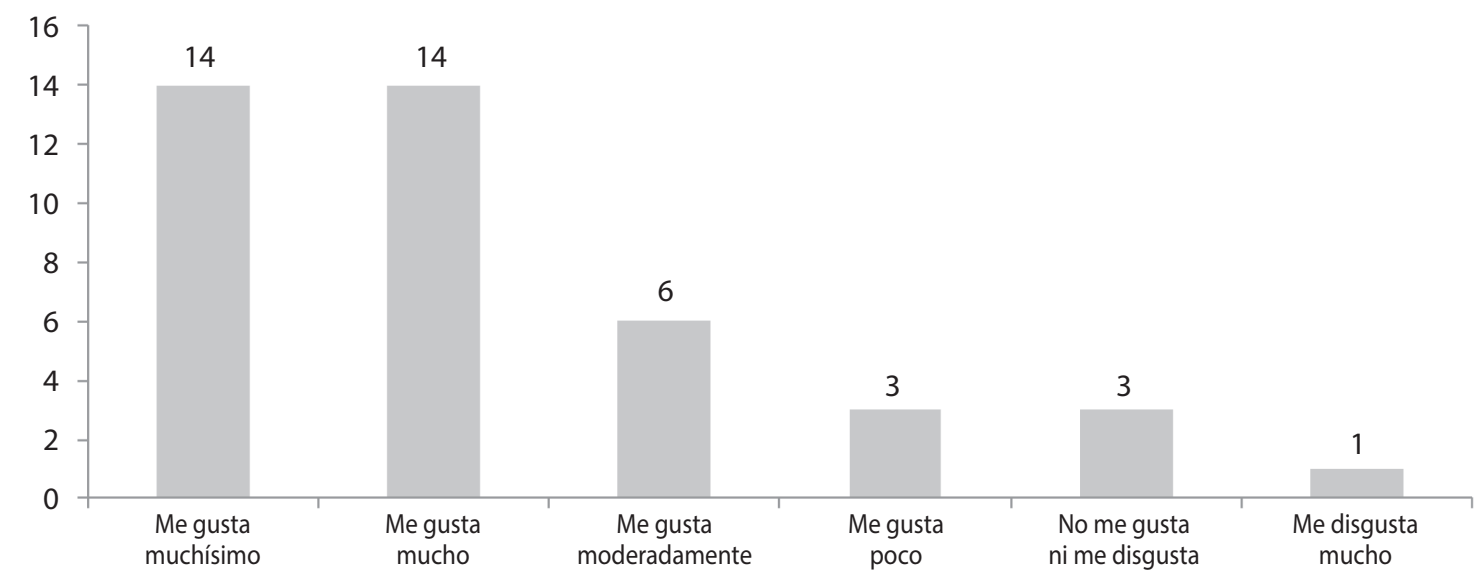

Figura 13. Grado de satisfacción de la cantidad de la textura del dip tipo hummus a base de gandul evaluada por 40 panelistas. Mediana 1 (estadística no paramétrica). Fuente: Autoras, 2013.

\section{Análisis estadístico}

\section{a. Prueba de normalidad}

La prueba de normalidad de Kolmogorov-Smirnov es significativa, por lo que las distribuciones de la variable no son normales y la estadística no paramétrica es la que se debe utilizar.

\section{CUADRO 6}

Prueba de normalidad para determinar la distribución de cada variable estudiada para en la prueba de grado de satisfacción del dip tipo hummus a base de gandul

\begin{tabular}{lccc} 
& \multicolumn{3}{c}{ Kolmogorov-Smirnov } \\
& Estadístico & gl & Sig \\
Sabor & .210 & 38 & .000 \\
Olor & .215 & 38 & .000 \\
Apariencia & .162 & 38 & .014 \\
Textura & .259 & 38 & .000 \\
Color & .152 & 38 & .026 \\
\hline
\end{tabular}

Fuente: Bermúdez, 2013

\section{b. Correlación de Spearman}

Dada la distribución no normal de los datos, se utilizó el coeficiente Rho de Spearman. Todos los coeficientes de correlación Rho de Spearman son positivos y altamente significativos $(\mathrm{p}<0.001)$, lo cual comprueba una asociación directa entre las cinco variables. 


\section{CUADRO 7}

Correlaciones de Spearman entre el contenido de sal, olor, sabor, textura y la apariencia del dip tipo hummus a base de gandul evaluada con una prueba de grado de satisfacción

\begin{tabular}{|c|c|c|c|c|c|c|}
\hline & & Sabor & Olor & Apariencia & Textura & Color \\
\hline \multirow[t]{3}{*}{ Sabor } & Coeficiente de correlación & 1.000 & $.490^{* *}$ & $.535^{* *}$ & $.535^{* *}$ & $.528^{* *}$ \\
\hline & Sig. (bilateral) & . & .001 & .000 & .000 & .001 \\
\hline & $\mathrm{N}$ & 41 & 41 & 41 & 41 & 38 \\
\hline \multirow[t]{3}{*}{ Olor } & Coeficiente de correlación & $.490^{* *}$ & 1.000 & $.572^{* *}$ & $.614^{* *}$ & $.471^{* *}$ \\
\hline & Sig. (bilateral) & .001 & . & .000 & .000 & .003 \\
\hline & $\mathrm{N}$ & 41 & 41 & 41 & 41 & 38 \\
\hline \multirow[t]{3}{*}{ Apariencia } & Coeficiente de correlación & $.535^{* *}$ & $.572^{* *}$ & 1.000 & $.788^{* *}$ & $.686^{* *}$ \\
\hline & Sig. (bilateral) & .000 & .000 & . & .000 & .000 \\
\hline & $\mathrm{N}$ & 41 & 41 & 41 & 41 & 38 \\
\hline \multirow[t]{3}{*}{ Textura } & Coeficiente de correlación & $.535^{* *}$ & $.614^{* *}$ & $.788^{* *}$ & 1.000 & $.571^{* *}$ \\
\hline & Sig. (bilateral) & .000 & .000 & .000 & . & .000 \\
\hline & $\mathrm{N}$ & 41 & 41 & 41 & 41 & 38 \\
\hline \multirow[t]{3}{*}{ Color } & Coeficiente de correlación & $.528^{* *}$ & $.471^{* *}$ & $.686^{* *}$ & $.571^{* *}$ & 1.000 \\
\hline & Sig. (bilateral) & .001 & .003 & .000 & .000 & . \\
\hline & $\mathrm{N}$ & 38 & 38 & 38 & 38 & 38 \\
\hline
\end{tabular}

**. La correlación es significativa al nivel 0,01 (bilateral). Fuente: Bermúdez, 2013

\section{c. Análisis de cluster de dos fases}

- Calidad de los conglomerados

Resumen del modelo

\begin{tabular}{|l|c|}
\hline Algoritmo & Dos fases \\
\hline Características de entrada & 5 \\
\hline Agrupaciones & 2 \\
\hline
\end{tabular}

Calidad de conglomerados

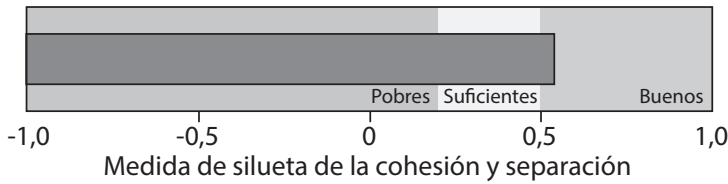

Figura 14. Calidad de los conglomerados generados para la evaluación del contenido de sal, el olor, el sabor, la textura y la apariencia del dip tipo hummus a base de gandul evaluada con una prueba de grado de satisfacción. Fuente: Bermúdez, 2013.

- Tamaño de los conglomerados
Tamaños de conglomerados

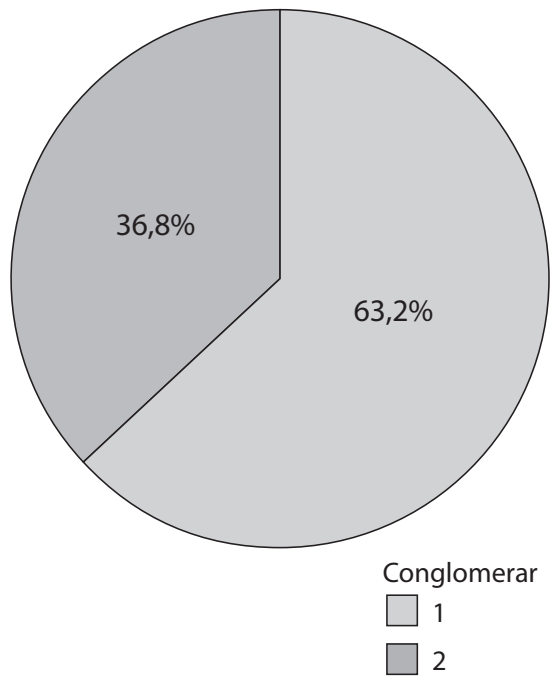

\begin{tabular}{|l|c|}
\hline $\begin{array}{l}\text { Tamaño del conglomerado } \\
\text { más pequeño }\end{array}$ & $14(36,8 \%)$ \\
\hline $\begin{array}{l}\text { Tamaño del conglomerado } \\
\text { más grande }\end{array}$ & $24(63,2 \%$ \\
\hline $\begin{array}{l}\text { Cociente de tamaños: } \\
\text { del conglomerado de mayor tamaño } \\
\text { al de menor tamaño }\end{array}$ & 1,71 \\
\hline
\end{tabular}

Figura 15. Porcentaje de panelistas que se encuentran en cada uno de los conglomerados formados estadísticamente para la prueba de aceptación realizada para el dip tipo hummus a base de gandul. Fuente: Bermúdez, 2013. 
Agrupaciones

Importancia de la característica

$\square 1,0 \square 0,9 \square 0,8 \square 0,7 \square 0,6 \square 0,5$

\begin{tabular}{|c|c|c|}
\hline Conglomerar & 1 & 2 \\
\hline \multicolumn{3}{|l|}{ Etiqueta } \\
\hline \multicolumn{3}{|l|}{ Descripción } \\
\hline Tamaño & $\begin{array}{c}63,2 \% \\
(24)\end{array}$ & $\begin{array}{c}36,8 \% \\
(14)\end{array}$ \\
\hline \multirow[t]{5}{*}{ Funciones } & $\begin{array}{c}\text { Apariencia } \\
1,79\end{array}$ & $\begin{array}{c}\text { Apariencia } \\
4,29\end{array}$ \\
\hline & $\begin{array}{l}\text { Olor } \\
1,54\end{array}$ & $\begin{array}{l}\text { Olor } \\
3,57\end{array}$ \\
\hline & $\begin{array}{c}\text { Textura } \\
1,58\end{array}$ & $\begin{array}{c}\text { Textura } \\
3,79\end{array}$ \\
\hline & $\begin{array}{c}\text { Color } \\
2,13\end{array}$ & $\begin{array}{c}\text { Color } \\
4,00\end{array}$ \\
\hline & $\begin{array}{l}\text { Sabor } \\
2,04\end{array}$ & $\begin{array}{c}\text { Sabor } \\
3,64\end{array}$ \\
\hline
\end{tabular}

Figura 16. Variable de mayor precedencia para la clasificación de los panelistas que evaluaron el dip tipo hummus a base de gandul por medio de una prueba de grado de satisfacción. Fuente: Bermúdez, 2013.

De acuerdo con los resultados anteriores, el primer grupo quedó conformado por la mayoría de panelistas (63\%) a quienes les gusta mucho el producto, y en el segundo grupo fue clasificado el restante $37 \%$ a quienes les gusta poco. La variable con mayor importancia (diferencia) para la clasificación es la apariencia y la de menor importancia fue el sabor. En el primer grupo el promedio global es de 1,8 y en el segundo de 3,8 .
Comentarios de los panelistas

CuAdro 8

Apreciaciones hechas por los panelistas sobre lo que les gustó y no les gustó del dip tipo hummus a base de gandul variedad criolla
Lo que les gustó

- Equilibrio del ácido

- La consistencia

- Sabor (16 personas)

- Buena presencia

- Color (2 personas)

- Textura (4 personas)

- Suave

- Apariencia (3 personas)

- Todo (3 personas)

\section{Lo que no les gustó}

- El olor (3 personas)

- Apariencia

- Falta de olores como el culantro

- Aguado

- Mucha sal

- Amargo (2 personas)

- Textura (3 personas)

- Sabor a ajo (2 personas)

- Color (2 personas)

- Sabor residual

\section{Discusión}

Valorando los resultados del panel sensorial de grado de satisfacción al que se sometieron 40 panelistas, podemos considerar que tanto la ensalada como el dip tipo hummus, fueron aceptados por los mismos. Para la ensalada se encontró un valor en la escala hedónica de 2.17 y si se compara con el cuadro 1 indica que el $45 \%$ de los panelistas se ubican en estas categorías (me gusta mucho-me gusta moderadamente). Para el dip tipo hummus, se encontró un valor de 2.47 y al comparar con el cuadro 5 se observa que el $53 \%$ de los panelistas se ubicaron en estas categorías (me gusta mucho-me gusta moderadamente).

Según Gacula \& Singh, 1984, las palabras o frases que describen cada nivel de la escala hedónica, reflejan las experiencias sensoriales de los individuos bajo una serie de condiciones sociales, ambientales, económicas y culturales, entre otras.

Si correlacionamos el resultado de la escala hedónica de la ensalada y el dip tipo hummus con estudios similares, podemos observar como 
los grados de satisfacción se pueden observar de manera objetiva, como por ejemplo en el caso de la caracterización de salchichas de tilapia con aceite y harina, que se valoró en tres tiempos diferentes, se encontró que las interacciones de aceite y harina, como la acción de estas como factores individuales no afectaron de manera significativa a los atributos sensoriales de color, textura y aceptación general, a través de todo el tiempo del estudio ( $\mathrm{P}>0.05)$. La aceptación general del producto cercano a "me gusta moderadamente", sin cambiar a través del tiempo de estudio. (Hinojosa e Intriago, 2012, p.8).

Seguidamente, para la evaluación de cada uno de los atributos de la ensalada con la escala hedónica (figuras 1 a 5), se encontró que para el sabor, acidez, cantidad de condimentos y sal, el $50 \%$ de los panelistas respondió un valor menor a 2 y el otro $50 \%$ respondió un valor mayor a 2 (mediana igual a 2), mientras que para el atributo de la apariencia, se encontró una mediana de 1.5, lo cual significa que esta característica tuvo una mayor aceptación que las demás. Lo anterior sugiere que se podrían mejorar aún más los cuatro atributos mencionados al principio.

Con respecto a la evaluación de los atributos del dip tipo hummus de Gandul (figuras 8 a 12), se encontró que para el sabor, el olor y textura, la mediana fue de 2; mientras que para la apariencia y el color la mediana fue de 3 . Lo anterior significa que estos últimos deben mejorarse, pues su valor se encuentra muy por debajo del esperado.

El análisis estadístico de los datos obtenidos para ambos productos mostró que se pueden describir mediante una estadística no paramétrica al no ser normales (ver cuadros 2 (ensalada) y 6 (dip tipo hummus) y por lo tanto nos dá una guía para saber el tipo de prueba al que se puede someter este producto.

La correlación de Spearman aplicada a los datos de ambos productos mostró que existe una relación altamente significativa entre las variables, lo cual significa que cada persona califica de manera similar todos los atributos evaluados.

Un estudio realizado por Leyva-Martínez, M; Pachón, H; Chaveco-Pérez, O; Permuy-Abeleira, N, Ferraz-Tellez, Y, Caballero-Espinosa, N y
García-Sánchez, E., 2010, sustenta lo encontrado en esta investigación, ya que al aplicar una prueba de aceptabilidad para evaluar sensorialmente el frijol común mejorado nutricionalmente (línea 38) y la variedad local (BAT 304) con productores y panelistas de dos comunidades de Holguín, Cuba, encontró que no hubo una diferencia estadísticamente significativa $(p=0,9)$ en el porcentaje de participantes que clasificaron la consistencia del caldo, el sabor del potaje y la textura del grano, considerando los criterios evaluados.

Contrario a lo anterior, en una investigación realizada por Centeno, P; Chow, Z; Cuadra, S; Brenes, D \& Pachón, H, 2011, se realizó una prueba de aceptación general sobre cuatro muestras de frijoles y se encontró que, "hubo diferencia estadísticamente significativa en el puntaje asignado a la aceptabilidad general de las cuatro muestras, así como para los atributos olor, color, sabor y textura (Tabla 1, p>0,05); estos oscilaron entre 2,5 y 3,0 .

Al realizar el análisis de conglomerados se logra apreciar que para ambos productos se obtuvieron dos grupos y de estos, el grupo con un mayor porcentaje de personas $(62,2 \%$ para la ensalada y $63.2 \%$ para el dip tipo hummus), indicó que le gustaba mucho, mientras que el grupo con un porcentaje menor de personas $(37.8 \%$ para la ensalada y $36,8 \%$ para el dip tipo hummus) mencionó que les gustaba moderadamente (ensalada) y poco (dip tipo hummus).

Si estos resultados los comparamos con un estudio realizado con arroz mejorado nutricionalmente con hierro y zinc, se encontraron resultados similares ya que la variedad IACuba, 30 tuvo una preferencia superior a 70\%. En las pruebas de aceptabilidad donde se evaluaron los parámetros olor, textura, sabor y color, los mayores porcentajes de preferencia recayeron en las opciones de "me gusta" y "me gusta mucho". Se debe destacar que la variedad obtuvo valores de aceptación superiores a $80 \%$ en las características evaluadas (textura, olor, sabor y color). (Puldón et al, 2011, p.8), podemos concluir en un comportamiento y por ende, resultados similares. 
Como siguiente paso en este estudio, se buscó cuales eran las variables de mayor y menor precedencia para cada producto. Para la ensalada, la de mayor precedencia fue la cantidad de condimentos (3.43) y la de menor precedencia fue la apariencia (1.52), lo cual significa que la cantidad de condimentos fue la variable que los panelistas objetaron más, mientras que la apariencia fue la cualidad más aceptada en el producto (ver figura 7).

En el cuadro 8, de acuerdo a lo anterior, se puede ver que en términos generales los panelistas comentaron que la apariencia fue un atributo que les gustó más en comparación con ciertos ingredientes como vinagre, cebolla y aceite, los cuales se adicionaron como condimentos y esto coincide con lo encontrado en el análisis de conglomerados. Cabe mencionar que en un focus group realizado para este mismo producto, los panelistas no expresaron disgusto por ninguna característica del producto, razón por la cual es importante realizar ambas pruebas antes de tomar una decisión de lanzamiento en el mercado (por publicarse Andrés \& Amerling, 2013).

Para sustentar lo anterior, en un estudio realizado acerca de las diferencias sensoriales que impacten en la preferencia de los panelistas de queso tipo "cuajada" elaborado con dos diferentes cuajos comerciales en Oaxaca, México, se encontró que los atributos sensoriales color blanco, grumoso en vista, textura grumosa al tacto, suave al tacto y presencia de suero fueron los responsables de las reacciones de preferencia en los panelistas. (López, G; Palomee, E; Carrasco, K; Mata, R; Castillejos, Z; Ramírez, E \& Cabrera, R., 2012, p.025), razón por la cual es muy importante la opinión que expresan los mismos.

Con respecto al dip tipo hummus, la variable considerada con mayor precedencia fue la apariencia (1.79) mientras que la cualidad que gustó más (menor precedencia) a los panelistas fue el sabor (3.64) (ver figura 15). Lo anterior coincide con los comentarios que realizaron los panelistas sobre este producto, ya que 16 personas mencionaron que el sabor del producto les gustaba, mientras que solamente una persona mencionó la apariencia como un atributo menos gustado (ver cuadro 8).

Un estudio similar se realizó para una cuajada pasteurizada, en la cual se quería determinar el efecto de dos tratamientos térmicos y dos concentraciones de cloruro de calcio en las características sensoriales de la misma, en tres tiempos diferentes. Los tratamientos preferidos por los panelistas para el día 1 y 8 fueron los elaborados con tratamiento térmico LTLT y el elaborado con tratamiento térmico HTST y $0.02 \%$ cloruro de calcio . El tratamiento menos aceptado fue el elaborado con tratamiento térmico HTST y $0.025 \%$. Los atributos que más influyeron en aceptación general fueron salinidad, sabor y apariencia. No se encontraron diferencias estadísticas entre tratamientos a través del tiempo (Cuellar, 2012, p. 9 y 10), por lo que podemos valorar cómo los atributos los percibe el panelista y expresa de esta manera su grado de preferencia.

\section{Conclusiones}

- La característica más gustada por los panelistas en la ensalada fue la apariencia.

- La característica más gustada por los panelistas en el dip tipo hummus fue el sabor.

- La cantidad de condimentos en la ensalada, es la variable que se debe trabajar más para ser mejorada.

- La apariencia y el color del dip tipo hummus es la característica a mejorar para este producto.

- Según la correlación de Spearman altamente significativa, cada persona califica de manera muy similar todos los aspectos evaluados para ambos productos.

- En ambos productos se encontró un grupo mayoritario que dijo gustarle mucho el producto $(62.2 \%$ para la ensalada y $63.2 \%$ para el dip tipo hummus), y un grupo minoritario que dijo gustarle moderadamente (37.8\% para la ensalada y $36,8 \%$ para el dip tipo hummus). 


\section{Recomendaciones}

- Realizar una correlación de los resultados obtenidos en esta investigación con los obtenidos en los focus group realizados para ambos productos y sacar conclusiones más firmes con respecto a su lanzamiento al mercado.

- Basado en los comentarios realizados por los panelistas, se recomienda valorar también el atributo "color" a la hora de evaluar la ensalada.

- Realizar pruebas para el mejoramiento del color del dip tipo hummus, por ejemplo, ya sea utilizando algún tipo de aditivo como la pimienta roja, o annato, pero que no tenga ningún efecto sobre el sabor.

- Realizar pruebas para valorar la cantidad de los condimentos que se adicionan a la ensalada (vinagre, cebolla, aceite) ya que estos no tuvieron aceptación por parte de los panelistas.

- Aplicar encuestas a los consumidores en general sobre el lanzamiento de ambos productos al mercado y sus posibilidades de aceptación.

\section{Referencias}

Andrés, C. (2012). Determinación de los descriptores sensoriales de un dip tipo hummus a base de Gandul (Cajanus cajan) mediante dos mini gropos focales. A publicarse en el 2013.

Andrés, C \& Amerling, C. (2013). Determinación de los descriptores sensoriales de una ensalada de Gandul (Cajanus cajan (L.) Millsp.) mediante un grupo focal. A publicarse en el 2013.

Anzaldúa-Morales, A. (1994). La evaluación sensorial de los alimentos en la teoría y la práctica. Acribia, Zaragoza.

Balluerka, N. \& Vergara, A. (2002). Diseños de Investigación Experimental en Psicología. Pearson, Madrid.

Centeno, P; Chow, Z; Cuadra, S; Brenes, D \& Pachón, H. (2011) Aceptación de tres líneas de fríjol (Phaseolus vulgaris L.) mejorado nutricionalmente por los consumidores nicaragüenses. Perspectivas en nutrición humana. 13(2): 179-189,. Recuperado de: http:// revinut.udea.edu.co/index.php/nutricion/article/ viewArticle/179

Cuellar Dole, (2012) Evaluación de dos tratamientos térmicos y dos concentraciones de cloruro de calcio en las propiedades físico-químicas y sensoriales de la cuajada. (Tesis). Zamorano, Honduras, Noviembre, 2012. pp. 1-31.

Delgado de la Torre, R. (2004). Introducción a la probabilidad y estadística. Editorial Madrid $1^{\circ}$ Edición. España.

Farfán, L. (2009). Aprovechamiento del suero dulce de queso campesino para la elaboración de una bebida refrescante con sabor a mora. Universidad Nacional Abierta y a Distancia "UNAD" Facultad de Ciencias Básicas, Tecnología e Ingeniería Programa Ingeniería de Alimentos Cead Zipaquirà, Cundinamarca.

Gallardo, G \& Renato, S (2011). Evaluación de cuatro niveles de materia orgánica en el cultivo de Amaranthus Caudatus e industrialización del grano para la comunidad de San Clemente - Imbabura (Tesis). Recuperado de http://repositorio.espe.edu.ec/ bitstream/21000/5112/1/T-ESPE-IASA\%20I-004589. pdf

Gastronomía y Compañía (s.f.). Hummus receta de puré de garbanzos. Recuperado de http:// ww w.gastronomiaycia.com/2008/01/10/ hummus-pure-de-garbanzos-delicioso-y-nutritivo/.

Hinojosa, J. \& Intriago, M. (2012). Evaluación de dos fuentes de carbohidratos y de grasa vegetal en la elaboración de un embutido a base de carne de tilapia negra (Oreochromis mossambicus). (Tesis).Honduras,2012. pp 1-32. Recuperado de: http://bdigital.zamorano.edu/ bitstream/11036/955/1/T3278.pdf

Leyva-Martínez, M; Pachón, H; Chaveco-Pérez, O; Permuy-Abeleira, N, Ferraz-Tellez, Y, CaballeroEspinosa, N \& García-Sánchez, E. (2010). Evaluación sensorial de frijol (Phaseolus vulgaris L.) mejorado nutricionalmente en dos comunidades cubanas. Agronomía Mesoamericana. 21(2). Recuperado de: http://www.scielo.sa.cr/scielo.php?pid=S1659$13212010000200007 \&$ script=sci_arttext

López, G; Palomee, E; Carrasco, K; Mata, R; Castillejos, Z; Ramírez, E \& Cabrera, R. (2012). Elaboración del queso fresco "cuajada" con dos tipos de cuajos comerciales: impacto sobre las reacciones de preferencia de 
los consumidores. Revista Venezolana de Ciencia y Tecnología de Alimentos. 3 (1): 014-027. Recuperado de: http://www.rvcta.org/Publicaciones/Vol3Num1/ Archivos V3N1/Lopez-Guzman_et_al._RVCTAV3N1.pdf

MAG (Ministerio de Agricultura y Ganadería, CR) 1991. Aspectos Técnicos sobre Cuarenta y Cinco Cultivos Agrícolas de Costa Rica. Dirección General de Investigación y Extensión Agrícola. San José, Costa Rica.

Mc Daniel, C. \& Gates, R. (2005). Investigación de Mercados. Thomson Editores, México.
Pedroza, H. \& Dicovsky, L. (2006). Sistemas de Análisis Estadístico con SPSS. IICA. Nicaragua.

Puldón, V; Suárez, E; Caraballo, R; Pachón, H \& Pompillo, C. (2011). Preferencia y aceptabilidad de la variedad de arroz IACuba 30 con alto contenido de hierro y zinc por mujeres gestantes en Cuba. Perspectivas en Nutrición Humana. 13(2): 123-124. Medellín. Colombia. Recuperado de: http://revinut.udea.edu.co/ index.php/nutricion/article/viewArticle/123

Rabello, E. \& Lima, E. (2005). Elaboración de barras de frutas deshidratadas a partir de banano y piña. (Tesis). EARTH. Costa Rica. 


\section{Apéndice 1}

Hoja de respuestas para realizar la prueba de grado de satisfacción de la ensalada a base de Gandul de 5 variedades. Fuente: Autoras, 2013.
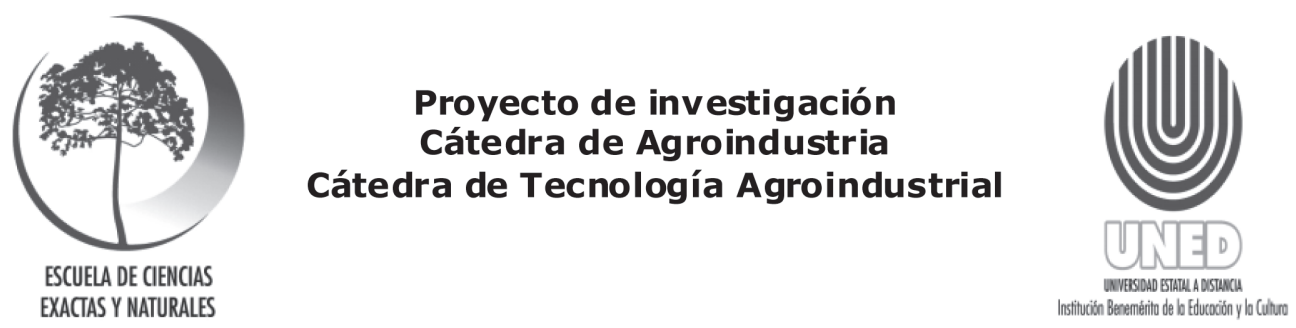

\section{Prueba de grado de satisfacción para ensalada a base de Gandul (Cajanus cajan)}

A continuación se le presentan 4 escalas hedónicas de 9 puntos para que califique la ensalada a base de Gandul que acaba de probar. Califíquela de acuerdo a sabor, acidez, apariencia, cantidad de condimentos y cantidad de sal.

Escala:

1 = Me gusta demasiado

2 = Me gusta mucho

3 = Me gusta modera damente

4 = Me gusta ligeramente

Sabor
$5=$ No me gusta ni me disgusta

$6=$ Me disgusta ligeramente

$7=$ Me disgusta moderadamente

$8=$ Me disgusta mucho

$9=$ Me disgusta demasiado

\begin{tabular}{|c|c|c|c|c|c|c|c|}
\hline$\Gamma$ & $T$ & 1 & $T$ & $T$ & $T$ & $T$ & $T$ \\
\hline 1 & 2 & 3 & 4 & 5 & 6 & 7 & 8 \\
\hline
\end{tabular}

$\underline{\text { Acidez }}$

\begin{tabular}{|l|lllll|l|l|l|}
\hline & 1 & 1 & 1 & 1 & 1 & 1 & \multicolumn{1}{l|}{} \\
\hline 1 & 2 & 3 & 4 & 5 & 6 & 7 & 8 & 9 \\
\hline
\end{tabular}

Apariencia

\begin{tabular}{|c|c|c|c|c|c|c|c|}
\hline Г & $T$ & $T$ & $T$ & $T$ & $T$ & $T$ & $T$ \\
\hline 1 & 2 & 3 & 4 & 5 & 6 & 7 & 8 \\
\hline
\end{tabular}

Cantidad de condimentos

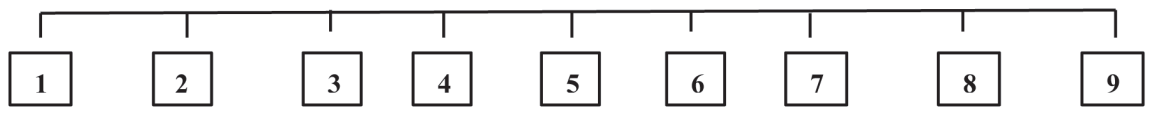




\section{Apéndice 2}

Diagrama de flujo del proceso de producción de la ensalada a base de Gandul. Fuente: Autoras, 2013.

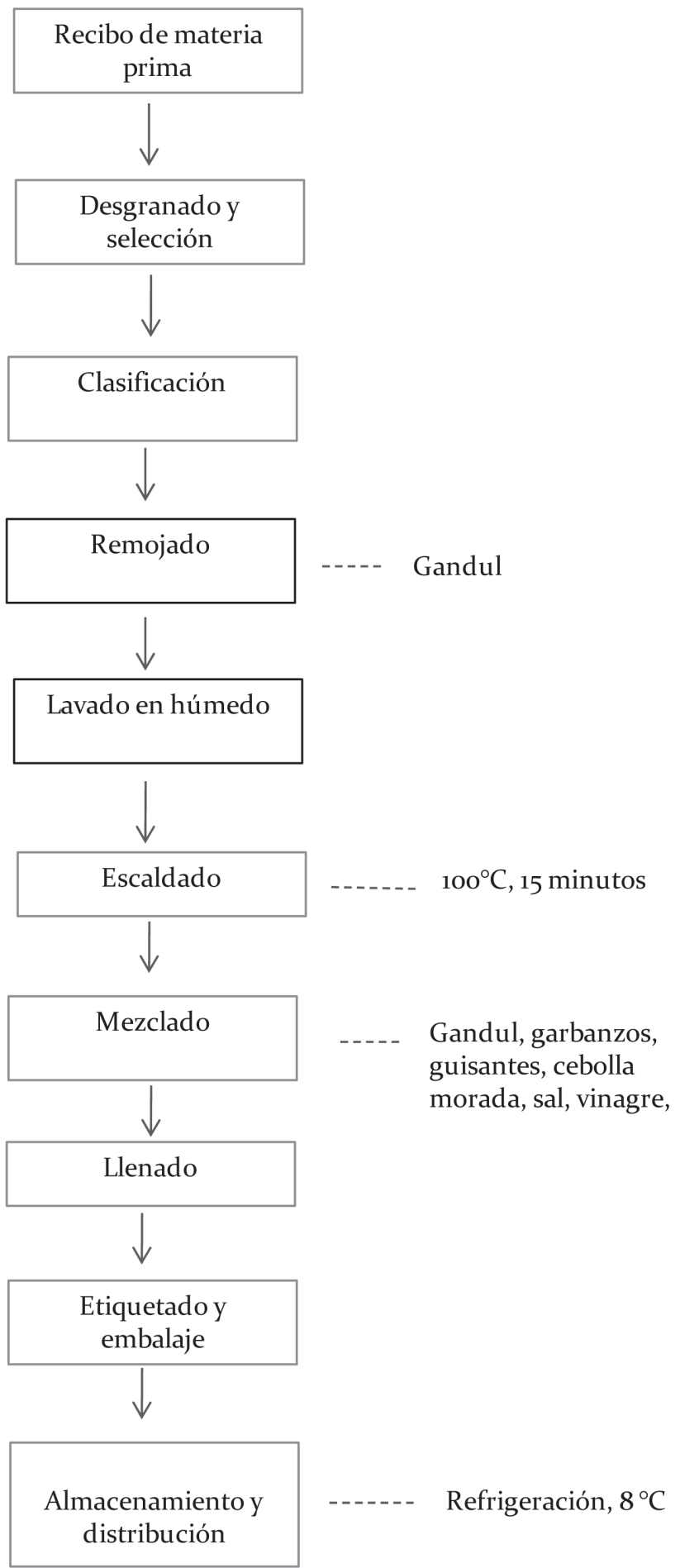




\section{Apéndice 3}

Hoja de respuestas para realizar la prueba de grado de satisfacción del hummus tradicional a base de Gandul criollo. Fuente: Autoras, 2013.

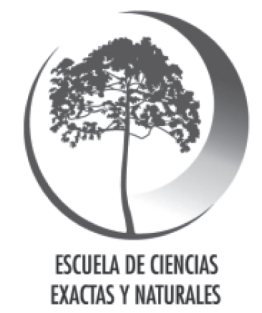

\section{Proyecto de investigación Cátedra de Agroindustria}

\section{Prueba de agrado para hummus a base de Gandul (Cajanus cajan)}

A continuación se le presentan 5 escalas hedónicas de 9 puntos para que califique el humus tradicional a base de Gandul que acaba de probar. Califíquela de acuerdo a sabor, acidez, apariencia, cantidad de condimentos y cantidad de sal.

\section{Escala:}

$1=$ Me gusta demasiado

$2=$ Me gusta mucho

$3=$ Me gusta moderadamente

$4=$ Me gusta ligeramente

$$
\begin{aligned}
& 5=\text { No me gusta ni me disgusta } \\
& 6=\text { Me disgusta ligeramente } \\
& 7=\text { Me disgusta moderadamente } \\
& 8=\text { Me disgusta mucho } \\
& 9=\text { Me disgusta demasiado }
\end{aligned}
$$

Sabor

\begin{tabular}{|c|c|c|c|c|c|c|c|c|c|}
\hline$\Gamma$ & 7 & $T$ & $T$ & $T$ & $T$ & \rceil & & $T$ & 7 \\
\hline 1 & 2 & 3 & 4 & 5 & 6 & & & & . \\
\hline
\end{tabular}

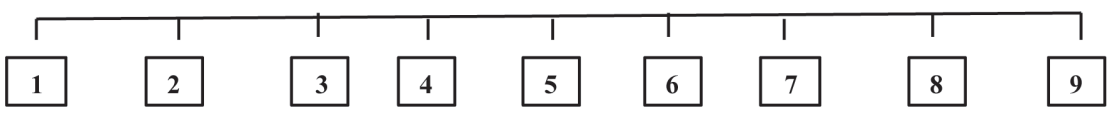

Olor

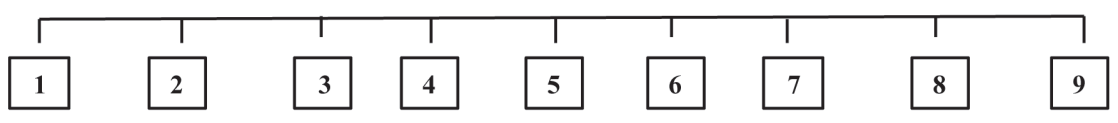

Apariencia

$\underline{\text { Textura }}$

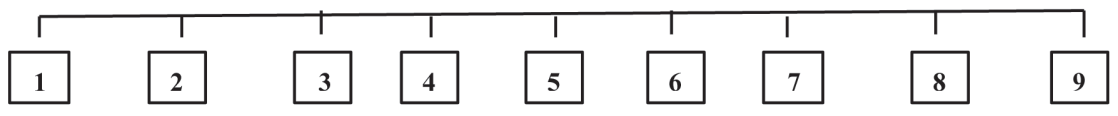




\section{Apéndice 4}

Diagrama de flujo del proceso de producción del hummus tradicional a base de Gandul. Fuente: Autoras, 2013.

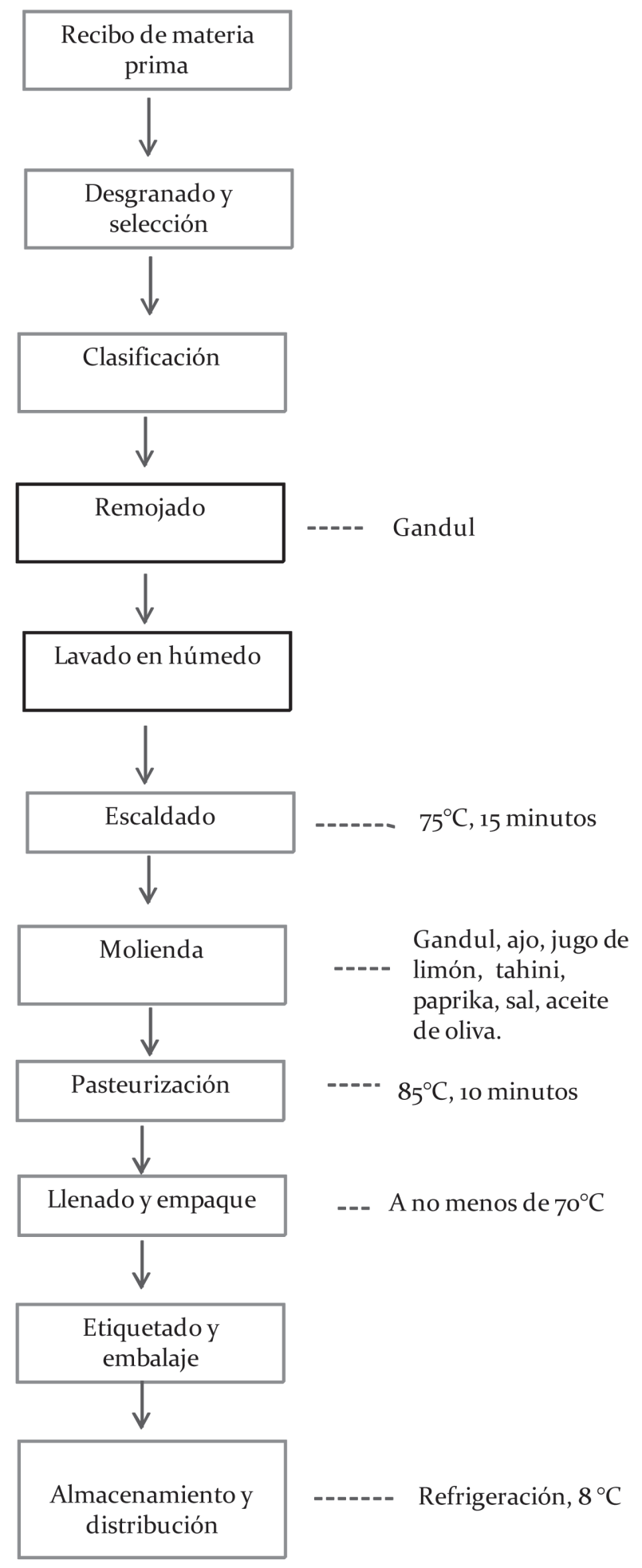


